\title{
Design and Development of an Inspection Robotic System for Indoor Applications
}

\author{
Pierluigi Rea, Erika Ottaviano
}

DICeM - Dept. of Civil and Mechanical Engineering, University of Cassino and Southern Lazio, via Di Biasio 43, 03043 Cassino (FR), Italy. Email: rea@unicas.it; ottaviano@unicas.it

\begin{abstract}
The inspection and monitoring of industrial sites, structures, and infrastructure are important issues for their sustainability and further maintenance. Although these tasks are repetitive and time consuming, and some of these environments may be characterized by dust, humidity, or absence of natural light, classical approach relies on large human activities. Automatic or robotic solutions can be considered useful tools for inspection because they can be effective in exploring dangerous or inaccessible sites, at relatively low-cost and reducing the time required for the relief. The development of a paradigmatic system called Inspection Robotic System (IRS) is the main objective of this paper to demonstrate the feasibility of mechatronic solutions for inspection of industrial sites. The development of such systems will be exploited in the form of a tool kit to be flexible and installed on a mobile system, in order to be used for inspection and monitoring, possibly introducing high efficiency, quality and repetitiveness in the related sector. The interoperability of sensors with wireless communication may form a smart sensors tool kit and a smart sensor network with powerful functions to be effectively used for inspection purposes. Moreover, it may constitute a solution for a broad range of scenarios spacing from industrial sites, brownfields, historical sites or sites dangerous or difficult to access by operators. First experimental tests are reported to show the engineering feasibility of the system and interoperability of the mobile hybrid robot equipped with sensors that allow real-time multiple acquisition and storage.
\end{abstract}

Keywords: Hybrid leg-wheel locomotion, Inspection, Interoperability, Low-cost monitoring, Experimental Tests.

\section{Introduction}

Inspection, assessment, and maintenance of the industrial sites and existing civil structures and infrastructure are current trends, including large-scale constructs such as plants, tunnels, bridges, roads and pipelines. Robots and automatic systems can perform the inspection process with objective results and high efficiency for those time consuming and repetitive tasks. They can also improve safety by performing inspection in dangerous or unsafe environments instead of the operators. Therefore, manual and (human) visual inspection are being replaced with more precise methods using mechanical, electronic and robotic systems. In addition, data provided by suitable sensors such as images, thermal images, laser can be combined with wireless communication and sensor network to constitute a powerful tool for inspection of industrial sites.

Robotics has been recently applied to tunnel inspection. In fact, tunnels (water supply, metro, railway, road) have increased in both total length and number, and will continue to do so. Robotic solutions were developed and used for inspection of tunnels [1-3] and pipes [4-6], involving visual inspections along with mapping, crack or deformation analysis, by using cameras, ultrasonic sensors, laser sensors, or even being able to perform cleaning or maintenance [7].

Robots were designed and used for search \& rescue and planetary exploration, as reported in [8-11]. Service robots can be used to autonomously execute guided inspection tasks in extensive industrial plants. If the equipment is arranged horizontally ground robots can be used, as reported in [12,13], in the case of vessels 
climbing robots are used[14].

Mobile robots have become a relevant research topic in recent decades and many researchers focus on the development of mobile robots, which are capable of performing tasks such as military operation, security monitoring, exploration, even in dangerous environment. In such situations, robots are mostly working in unstructured environments, which means that the information of the environment where the robot works is unknown. Therefore, in this case the obstacle avoidance is the most important feature for a mobile robot and that is why most researchers focus on the study of the robot with good terrain adaptability. Moreover, such characteristic makes the robot robust and capable to carry in a stable way suitable instrumentation for the given task. Recently, due to the catastrophic events of the earthquakes in center of Italy in 2009 and 2016, the inspection task is the most common and requested activity that a mobile robot has to perform in unstructured environment, in unsafe and non-planned events.

Mobile robots are classified as belonging to either legged, or wheeled/tracked, or hybrid mobile systems [15] according to their locomotion systems. Wheeled systems are the most efficient, energy saving and reliable solutions for both ground and climbing mobility, in the latter case the actuation system can be of magnetic or pneumatic type to provide adhesion to the wall to climb. Wheeled robots mainly get the best performances in terms of robust motion on flat surfaces, but they are almost ineffective in overpassing obstacles or on uneven terrain. Tracked systems can partially solve the problem, but they cannot overpass obstacles greater than the track high. Theoretically, legged robots are the solution to all the abovementioned problems [16], but they have to deal with important issues related to energy consumption, stability and locomotion speed, either if the robot has two, four, six or more legs $[17,18]$. Hybrid mobile robots move thanks to the combined action of wheels (or tracks) and legs. Examples of such systems are [1924]. There are four possible combinations of locomotion types that lead to hybrid systems, namely legswheels, legs-tracks, wheels-tracks, and legs-wheels-tracks. The three main categories and the four hybrid categories of ground mobile robots are described in [25]. Another classification deals with the combination of locomotion types, i.e. in series or in parallel. In the first type legs ( or sub-tracks) provide traction or obstacle avoidance and act in parallel to wheels. In the second type, legs and wheels are coupled in series to operate together. The first type is chosen as design solution for a large number of prototypes and it is used in this context for the proposed system.

Interesting devices have been developed for automatic inspection; systems are equipped with sensors allowing them the exploration of a building in total autonomy, as reported in [26]. In most of cases the sensor suite is devoted to SLAM, automatic path following, obstacle detection and avoidance. Our aim is to test lowcost technologies to monitor and manage sites of interest, trying to significantly reduce the acquisition and maintenance costs and the time needed to the relief.

In addition, inspection and monitoring systems are only apparently tools easy to use and manage, in fact, they hide drawbacks such as high purchase and maintenance costs as well as significant financial commitment related to data management and processing. Those factors greatly influence the wide spreading of those systems, which use is usually limited to high relevance applications, such as military, or in case of disasters like at Fukuhima nuclear plant in 2011 [20].

In order to enhance the use and the spreading of technologies, new solutions have been explored dealing with the concept of robotic and automatic survey using low-cost technology. More specifically, the use of a robotic platform may drastically reduce the time and cost needed for a relief, if compared to a classical approach.

In addition, teleoperation allows the inspection of sites dangerous or difficult to access by human operators. Moreover, the use of low-cost technology both for the mechanical design solution of the mobile robot and the onboard sensors allow the substitution of the IRS in the case of damages or if the robot is lost. 
The paper is organized as follows: Section 2 outlines requirements for automatic/robotic inspection and monitoring in industrial environment. A solution is proposed as based on a hybrid mobile robotic platform. Section 3 presents the mechatronic design of the proposed system with suitable sensorization and Section 4 proposes first experimental results for the performance assessment. Finally, conclusions are outlined.

\section{Requirements and solutions}

In the following, the task requirements and the proposed robotic platform will be illustrated and discussed according to the inspection and monitoring tasks in industrial environment. Nevertheless, the considerations and proposed solutions can be broadly adapted to different types of scenarios.

\subsection{Task requirements}

The tasks need to accomplish some basic requirements in terms of capabilities, such as mobility, sensorization, communication, and hardware and software reliability. Those issues are detailed in the following for the inspection tasks both in industrial and non-structured environments.

Mobility issue. Urban search \& rescue, indoor inspections in industrial environment and outdoor inspections due to planned or catastrophic events, and military intelligence have one need in common, the need of a small sized mobile robot that can travel across a large variety of scenarios. Some of these indoor or outdoor areas to access are not only difficult to reach, but may present also safety and health hazards to human inspectors. Therefore, the development of robots with high mobility and compact design is a priority to cope with these scenarios. Desired capabilities for such robots are: 1) the ability to traverse uneven terrain, slopes, steps, both indoor, in the worst conditions with obstacles such as concrete floors cluttered with debris, unfinished floors such as those found on constructions sites, or obstacles to surpass or circumnavigate, or outdoor in the presence of rugged terrain with stones or plants. Another ability is 2) to fit either through small openings, or overpass wide gaps; 3 ) the ability to climb up and over vertical steps or overpass obstacles; 4) the ability to travel inside and outside of horizontal, vertical, or diagonal pipes such as electric conduits or water pipes. Those high varieties of areas and circumstances make the mobility issue a prior requirement for such type of mobile robots application. Therefore, the robotic mobile structure has to fulfill the requested mobility issues according to the scenario and the task.

Sensorization. Sensors suite for mobile robots can be classified in two types, namely internal/navigation sensors and external/application sensors. The internal ones give the robot mobility control and navigation capabilities. They may include such devices that allow the robot to be commanded and controlled, i.e. activate and control the motors, and equipment for localization, obstacle detection, position estimation and goal tracking. These sensors are encoders, proximity sensors, GPS, accelerometers, gyroscopes, magnetic compasses, tilt and shock sensors. External sensor suite is related to the specific task, if the inspection/surveillance/search and rescue task is the goal, then sensors such as cameras, thermal cameras, laser, light, temperature, gas, smoke, oxygen, humidity, listening and ultrasound are the most common used sensors to detect the environment in indoor and outdoor environment.

Communication tools. Either wireless or wired-based communication systems can be used for robot localization/navigation and data transmission. Therefore, in indoor environment the condition of signal reception inside a building needs to be checked. In the case of poor or bad reception, a wired-only communication solution has to be considered in order to ensure reliable communication during the inspection.

Hardware and software reliability. It is necessary to specify, at early design stage, if engineers, who are familiar with the design and development of the mobile robot, will operate it; or if novice operators, who are not necessarily capable to perform maintenance to the robot or internal and external sensors suites, will use 
it. These situations influence both hardware and software reliability. For the first issues, robust hardware has to be designed, built and provided in order to be able to perform a mission without the need of robot maintenance due to breaks or failure circumstances. Referring to the software reliability, it must be easy-touse and robust, and has to provide almost autonomous data acquisition. In many cases, the engineers are the operators that do the task, but in industrial planned inspection task it would be preferable to be able to adopt the second choice to widespread the use of the proposed solution.

Navigation modes. Three levels of autonomy can be defined: pure teleoperation, safeguarded teleoperation, and autonomous navigation. In teleoperation mode, imagery can be transmitted to the user over either the analog video transmitter or radio modem. In safeguarded teleoperation mode, an onboard obstacle avoidance behavior modifies the users teleoperation commands via an arbitration scheme. In autonomous navigation mode, obstacle avoidance is combined with onboard goal seeking behaviors (waypoint following) that move the robot toward the desired target. For the inspection and monitoring tasks, in this paper we have considered the pure teleoperation mode. Nevertheless, the safeguarded teleoperation mode is under experimentation and can be considered as enhancement of the robot's capabilities.

\subsection{Mechanical architecture of THROO}

In order to accomplish the proposed task, the THROO (Tracking Hybrid Rover for Overpassing Obstacles) in Figure 1a) has been used as mobile platform equipped with the tool kit for inspection. According to the authors' knowledge, the most efficient systems for obstacle climbing and obstacle overpass are hybrid solutions dealing with tracks and sub-tracks.

The design concept of the THROO hybrid rover prototype is shown in Fig. 1 with low-cost easy-operation features. In order to obtain these characteristics for a ground mobile robot, requirements have been considered such as compactness, lightweight, reduced number of DOFs. The leg design shown in Fig.1b) has 1-DOF giving the possibility to combine more legs sharing the same input crank. This solution allows the use of just one actuator for operating at same time four legs in order to obtain a robot capable of overpassing a large number of obstacle types [27]. More specifically, according to the 3D scheme of the legs in Fig. 1a) and their actuator, a common shaft for left and right pairs of front and rear legs is connected to the motor through a belt transmission. The common shaft directly connects the left and right cranks at points $M$ and $\mathrm{M}^{\prime}$. In the planar scheme in Fig. $1 \mathrm{~b}$ the crank is drawn in black and indicated by $\mathrm{B}_{0} \mathrm{~B}$. The four-bar linkage for each leg is labeled $\mathrm{B}_{0} B A A_{0} P$, being $B_{0}=M$. According to the proposed mechanical design, front and rear legs share the same input crank $\mathrm{B}_{0} \mathrm{~B}$. It is worth noting that left and right arrangements of the legs are symmetric with respect to the sagittal plane, therefore the robot has four legs, each of them being the four 1-DOF linkages.

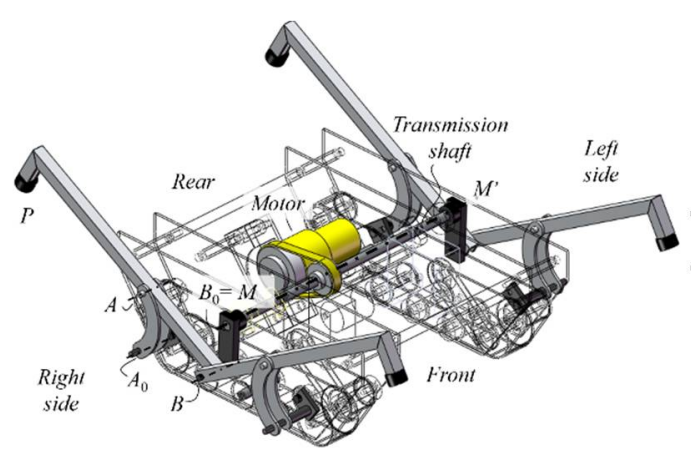

a)

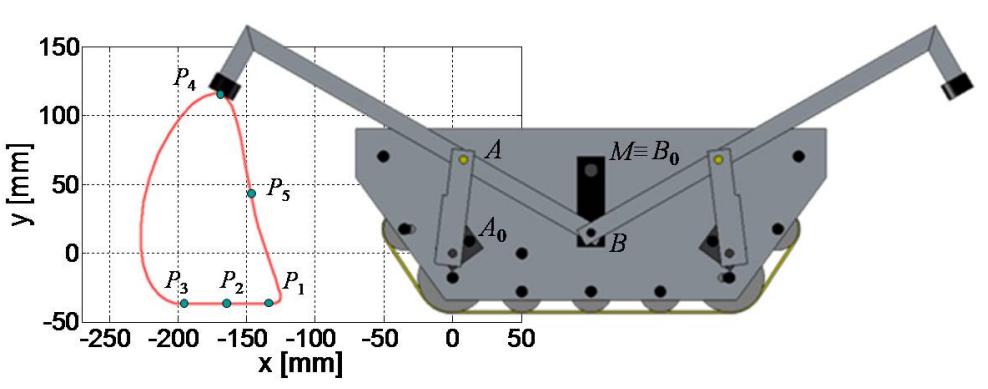

b)

Figure 1. THROO mobile robot: a) a prototype; b) schemes of the legs and their trajectory. 
In particular, a 1-DOF linkage is used to get a suitable trajectory of the leg tip to aid the rover with rear and front legs to overcome obstacles. The synthesis procedure was based on Burmester problem to obtain the leg mechanism according to design specification and it was reported in [27].

In particular, the five given poses $\mathrm{P}_{1}$ to $\mathrm{P}_{5}$ in Fig. $1 \mathrm{~b}$ ) are used to solve the synthesis problem. Design solutions involving a higher number of DOFs may be also used to increase the robot flexibility in overcoming obstacles, as it is shown in $[28,29]$, but they will increase the robot complexity and make the control strategy more complex. Therefore, in this context, the legs design has been based on the concept that they are in contact with the ground/obstacle through the leg endpoints, and they have been designed to get a suitable motion to approach the obstacle and overpass it, but do not interact with ground on flat terrain, as shown in the scheme of Fig.1b). Simulations of the Dynamics of the robots on uneven terrain have been carried out, as reported in [27]. Experimental tests have been also carried out. As the outcome of the experiments the built prototype can climb up to an obstacle that is $125 \%$ of the track high, the maximum tilting angle is 43 deg and its maximum value before overturning is about $60 \mathrm{deg}$. It has been verified that the legs can be used to stabilize the hybrid robot during the motion and prevent longitudinal and lateral overthrow in climbing obstacles [27]. Two operating modes are possible: 1) rover-like motion on flat or smooth terrain, which is the most efficient type of locomotion when allowed; 2) walking-like motion, when approaching and overcoming obstacles. In particular, it is worth to note that the symmetrical arrangement of front and rear legs has been adopted for several reasons [27]: I) stabilization of the robot in terms of overturning; II) possibility in almost all cases to change the direction of motion, when it is easier than turn; III) when the front (rear) legs approach the obstacle, the rear (front) leg are used as propulsive elements to help the robot in overcoming the obstacle.

The robot specifications are the following: 3 Degrees Of Freedom (DOF) one for rear and front legs and two for the tracks; robot size (in mm 300) (legs extended 550) X 420 and height 140 (legs extended 550); weight $4.5 \mathrm{~kg}$ without batteries. The tracks are made of rubber/aluminum alloy and are $260 \times 280$, the legs dimensions are $272 \times 10$. The travelling performance are the following, max speed $0.5 \mathrm{~m} / \mathrm{s}$; max step 100 $\mathrm{mm}$; max slope $60 \mathrm{deg} ; 2$ hours of autonomy. The actuation system is composed by $2 \mathrm{DC}$ motors for the track with following features: power supply of $24 \mathrm{~V}$, max torque of $5 \mathrm{Nm}$, nominal power of $3.9 \mathrm{~W}$. The motor used to actuate the legs has a power supply of $24 \mathrm{~V}$, max torque of $12 \mathrm{Nm}$, nominal power of $24 \mathrm{~W}$.

\section{Mechatronic design of the Inspection Robotic System (IRS)}

Two main parts, the first one devoted to the robot control for mobility issues and operation modes, and the other part responsible to manage the external sensor suite, data acquisition and storage compose the mechatronic design of the Inspection Robotic System (IRS). In the following, the robot control is described first, and then the sensorization for the proposed application. Finally, the control scheme is detailed for the IRS mechatronic system composed by robotic mobile platform and its sensor suites.

Figure 2 shows a scheme for the control of the THROO. According to the scheme reported in Figure 2, (1) represents the tablet with the software installed onboard for the robot motion control and navigation. More specifically, the DENSION WIRC software allows to turn on and off digital inputs and outputs, and allows the robot to move forward (F) and backward (B), turn right (R) and left (L), thanks to the two sliders represented in the zoomed view in (7). The USB WiFi router type TP-LINK Model TL-WN821N is indicated with (2) and allows the tablet to access and connect to the WIRC hardware. The webcam named as CAM (3) is Logitech U0024 type and the target/vision is sent and displayed on the tablet. The DENSION WIRC hardware (4) provided by four digital Inputs, four Digital Outputs and eight channels, is used to control the Servo Motors. The hardware in (4) is connected to and command the Arduino board (5), which drives via relay (6) the robot's actuators. The target (8) is displayed on the tablet and finally (9) represents the overall mechatronic system architecture for the robot navigation. 


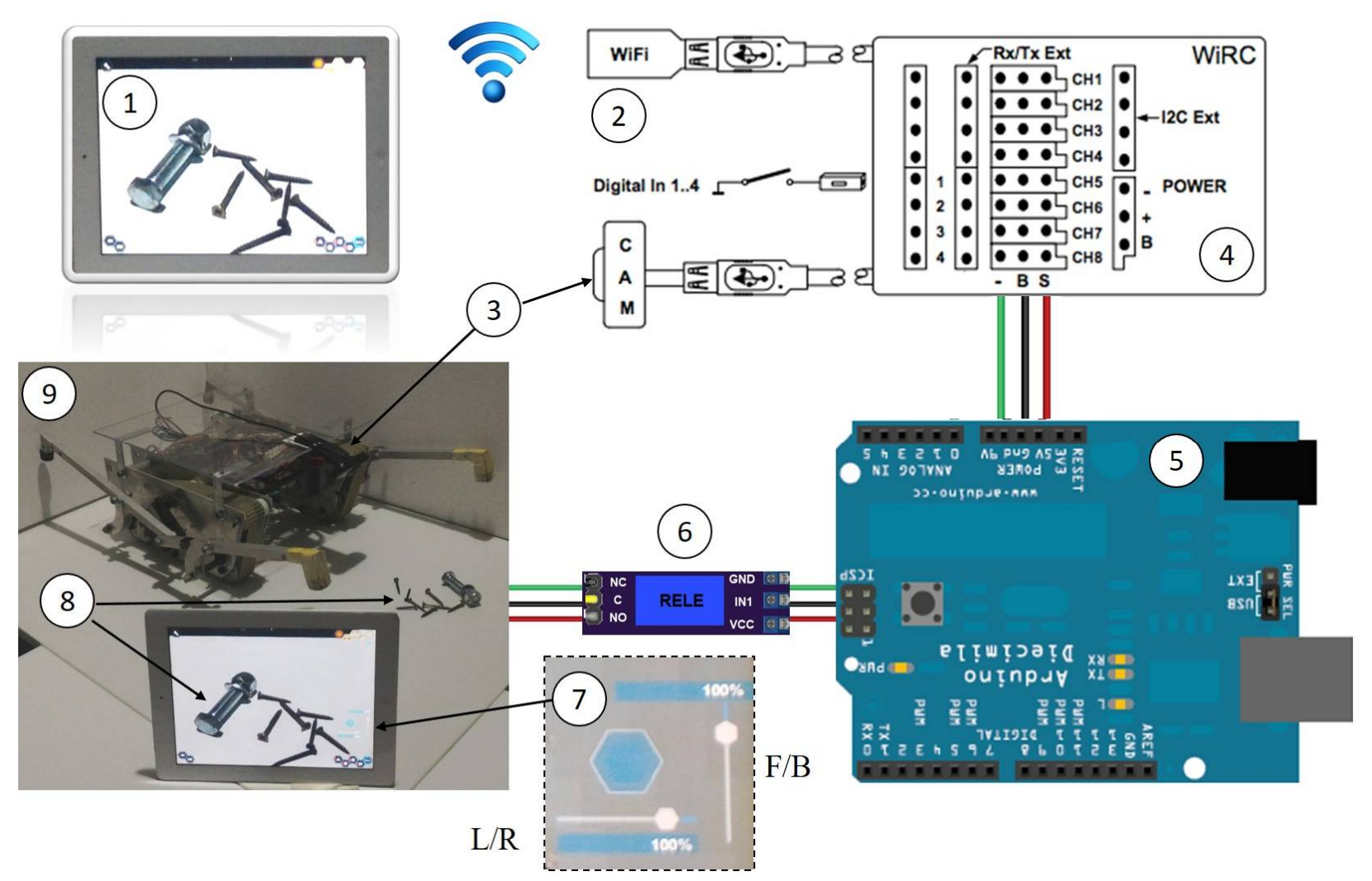

Figure 2. Mechatronics architecture of the control for the THROO robot in Fig.1.

The navigation sensor suite was driven by the need for daylight obstacle detection, mapping, position estimation, and tracking. Sensors devoted to the specific application are both external and internal ones [30]. Thus, when the robot moves, the navigation sensor suite also named internal sensors suite acquires information sent to the tablet for the teleoperation making the operator capable to understand the environment and presence of obstacles. External sensors suite is used for the inspection and monitoring tasks. More specifically, referring to Figure 3,1 ) is the FLIR ThermaCAM S40 thermal infrared camera for videos, and 2) is an electronic board equipped with accelerometer, gravity and gyroscope sensors, GPS sensor, magnetic field and acceleration sensors. The front camera view for navigation is displayed as 3 ). The 3D scan is the Xbox Kinect shown in 4), provided with an infrared sensor, and two additional micro cameras.

Although the selected navigation mode is tele-operated, the sensor suite allows the system to be integrated with further abilities of semi-autonomous or autonomous operation, which will be considered as future development of the system.

In order to manage external and internal sensorization, the overall layout for the ISRS is shown in Figure 4, in which main components may be recognized.

Based on the information received, the measured data are displayed on the tablet (3) and laptops (4) and (5) (dual display system version). In particular, the navigation control is operated by the tablet, and data sensors are displayed on laptops 4) and 5).

In particular, in Figure 4 no. (1) is the heat source that is scanned by the thermal camera; (2) is the robot performing the monitoring task and it is equipped with sensors onboard. The robot is tele-operated and controlled by the tablet in (3). Two laptops are used for displaying purposes, although it is not necessary because all data from sensors are stored on a PC and then in a Cloud, as described in [31]. More specifically (4) is used to display the thermographic camera outcome.

The laptop (5) is used for viewing in real time the 3D scanning (a) and a display screen (b) with information related to the robot itself, namely GPS, accelerometers, inclinometer, which are useful for navigation. More 
specifically, zoomed views of the displays are reported in Figures 5a) and 5b).

It is worth noting that this is a layout to show and describe the behavior of the system. In the set-up for monitoring and experimental activities, most of the sensors must be protected against dust, dirty and possible damages by using a cover. Such cover is not displayed.

Figure 6 shows the scheme for the control architecture used to operate the THROO robot and interoperation with the equipment on-board. The laptop (5) in Fig.4 is connected via USB to the control board and is used to upload the control algorithm on board to Arduino. The Arduino board that runs the control algorithm operates the three DC motors that handle omnidirectional motion, and drives legs.

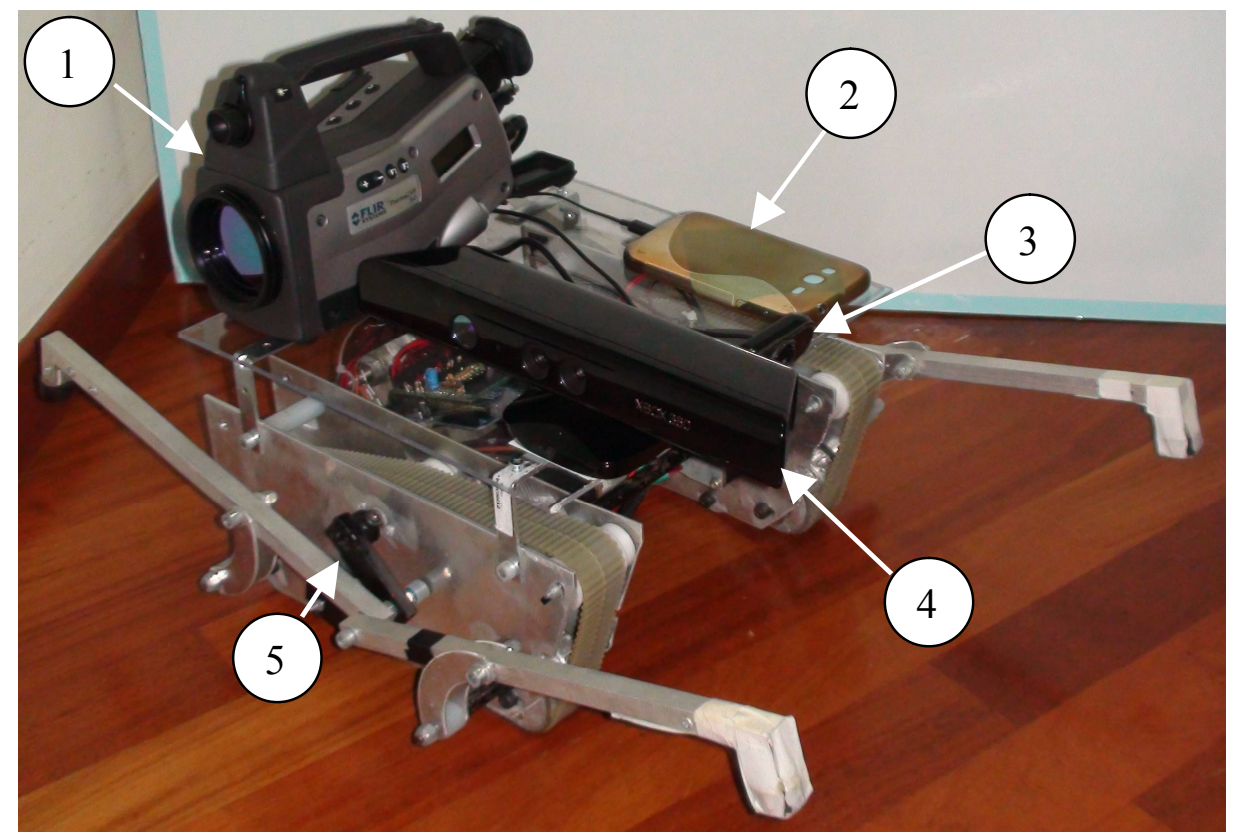

Figure 3. Sensors installation on the robot.

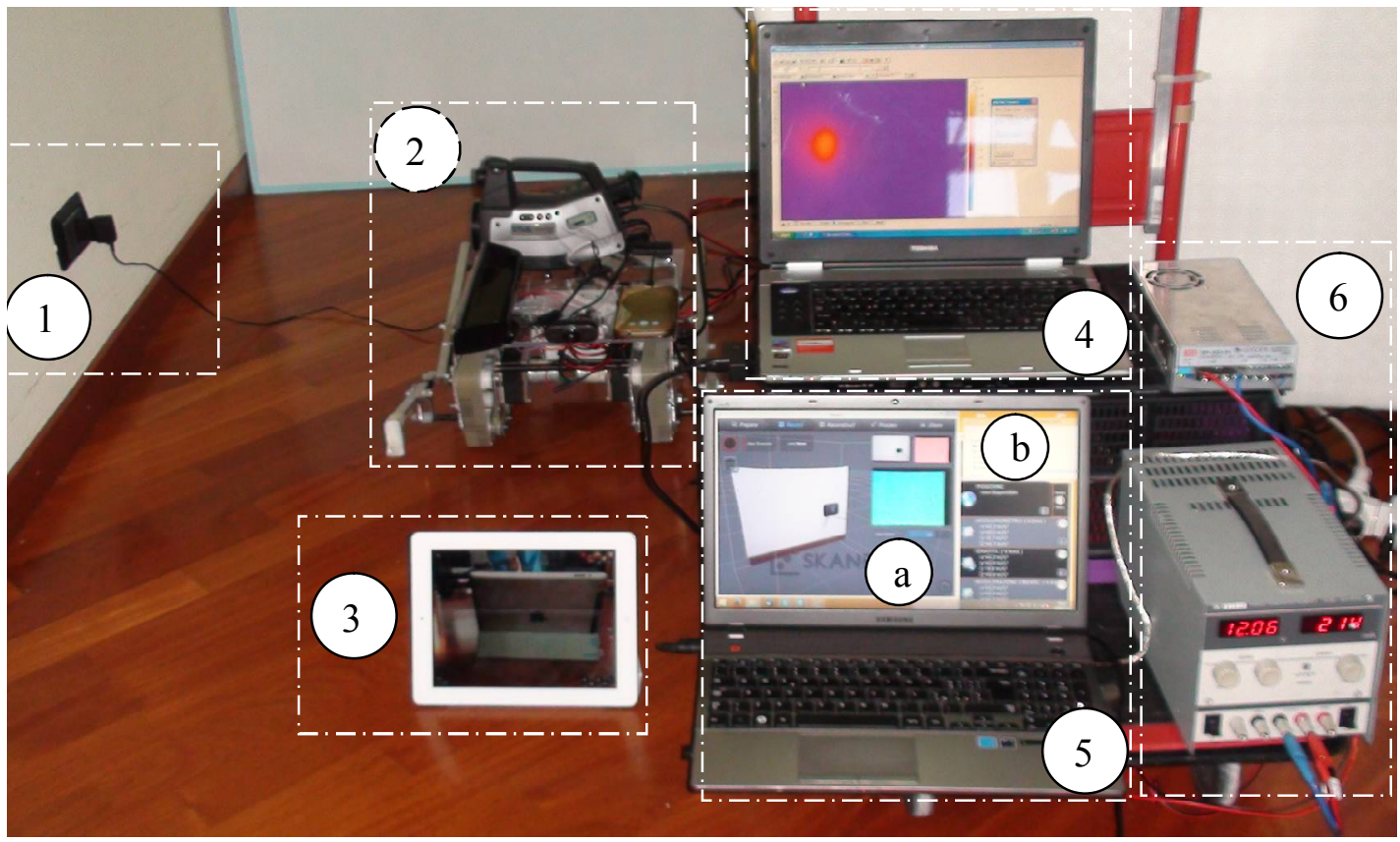

Figure 4. Overview of the proposed mechatronic system. 


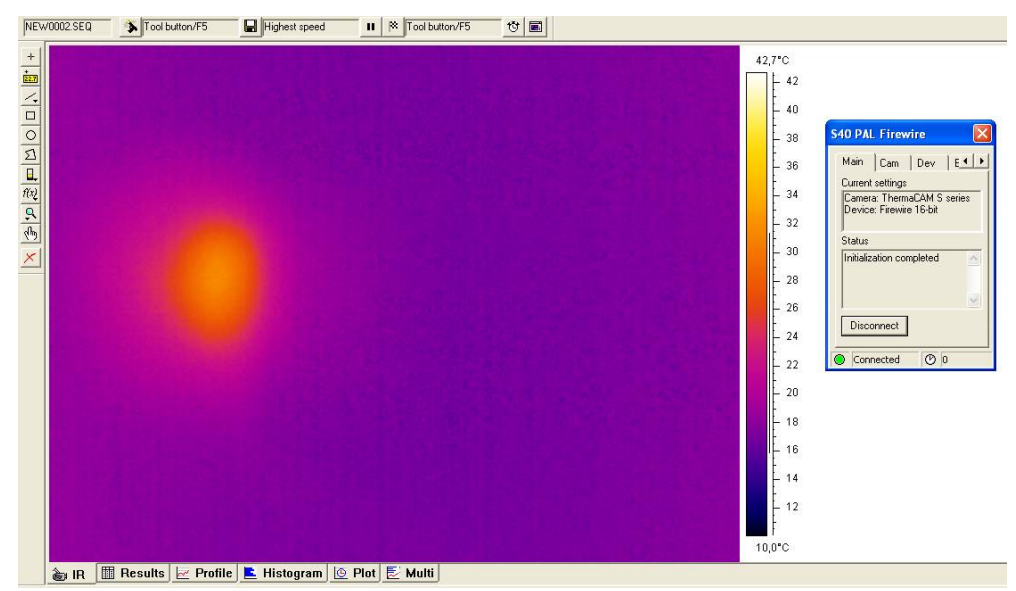

a)

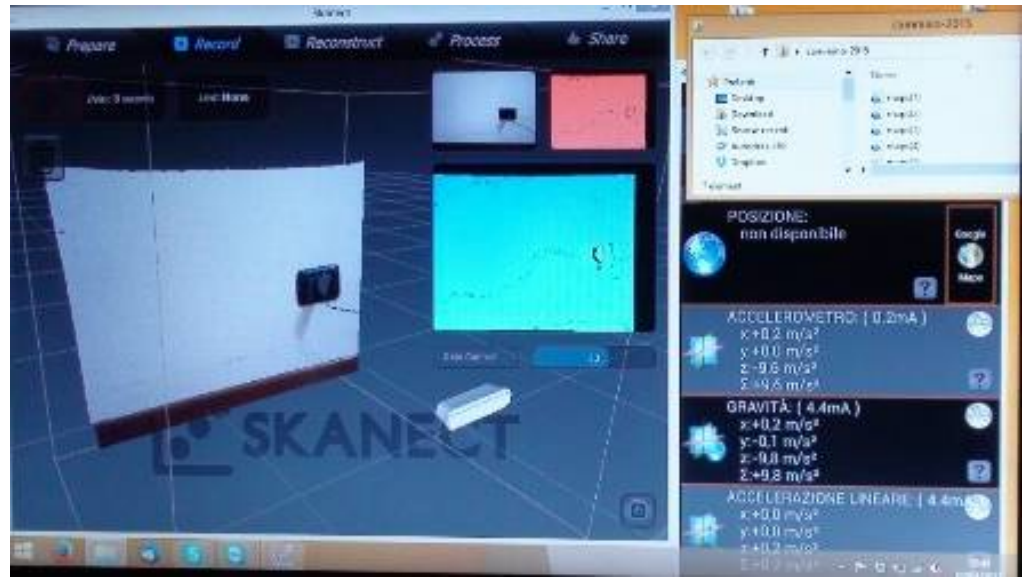

b)

Figure 5 Overview of the displays (4) and (5) in Fig. 4 for the proposed mechatronic installation: a) the thermal infrared detection display; b) 3D Scan display and electronic board sensors display.

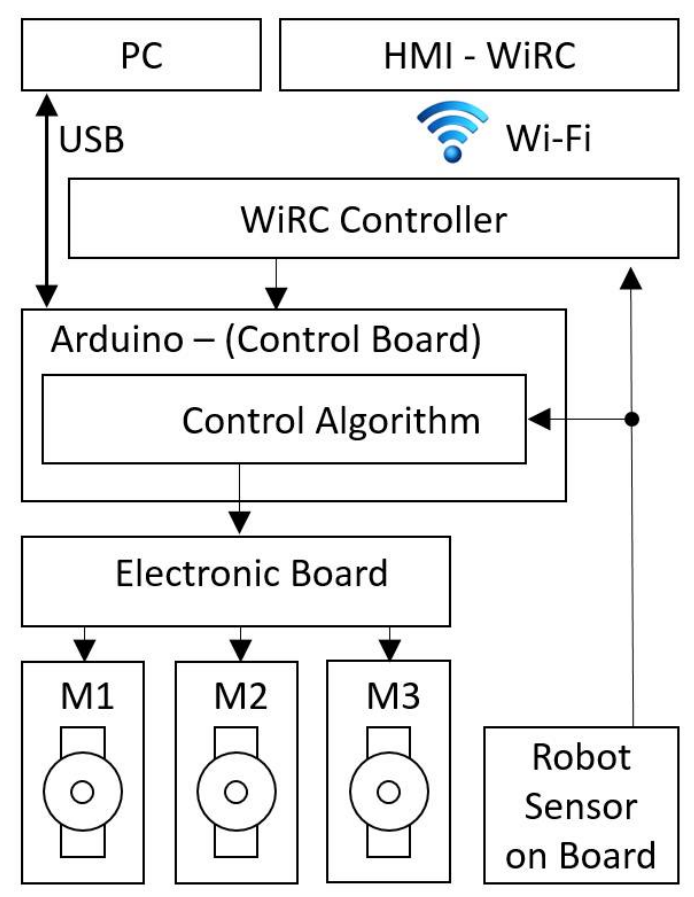

Figure 6. A scheme for the implemented control architecture. 
The interoperability of the sensors on board with navigation sensorization is managed by the control board and the WIRC Controller. Particular attention has been devoted to design the interface control between the WIRC controller and Arduino board, which is managed through digital signals modulated in PWM (PulseWidth-Modulation). A constant train of pulses, all having the same duration and the same frequency, constitutes the carrier wave. When this signal is modulated it transmits a value proportional to the control signal (the position of the slider on the tablet), therefore the modulator has the task of varying the duration of the carrier wave pulses directly proportional to the amplitude of the reference signal.

\subsection{Sensors description}

Inspection and surveillance of industrial sites, structures, and infrastructure are important issues for their sustainability and maintenance. These indoor and outdoor operations are usually performed by inspectors and requires accomplishing standards, and are time consuming. In addition, these environments are usually characterized by dust, humidity, and in some cases by absence of natural light. Therefore, classical approaches that rely on large human activities are performed in unsafe or dangerous conditions in some particular cases. In recent years, several studies have been conducted to explore the use of robotic systems to perform tele-operated monitoring and inspection to reduce human efforts. In particular, in industrial and outdoor applications, automation and robotics can be an efficient solution that may help.

Visual inspection is probably the most important among the others in indoor and outdoor inspection. Unlike 2D map building, 3D mapping can determine the size of object and reconstruct the environment. Thanks to the improving computing performances and development of measurement sensors, 3D map building research has greatly increased. The used devices for obtaining 3D information are laser scanners, and ToF (Time of Flight) cameras to produce the point clouds of the environment or the object. Vision SLAM extracts features from the camera image and estimate motion. Referring to 3D mapping and SLAM, a number of authors in very recent years explored the possibility to use a Microsoft Kinect sensor to identify features of an object or the environment, as for example $[32,33]$. The Kinect sensor is also called RGB-D camera and includes a color camera, a depth camera, an accelerometer, and tilting motor. The depth sensor is a near infrared projector that projects a known structured pattern being observed by an IR camera. Indeed the RGB D camera reconstructs a 3D map by combining the color information and distance information.

In this context, experimental tests have been carried out in laboratory environment, an example of the outcome is shown in Figure 7 by using a commercial software for 3D map building, in which the StL file is displayed of the 3D Scan for a mechanical component. These experiments have been carried out in order to test the reconstruction capabilities of the system architecture for the proposed application.

For many industrial applications, not only for monitoring and surveillance but also in production lines of electronics and automotive, obtaining thermal data is of great interest and crucial importance, because it can provide different and complementary information to vision. Thermal imagery provides an excellent solution in industrial environment for applications involving non-contact precision temperature measurement and non-destructive testing [34]. In addition, it may be used in other environments, as for example brownfields of historical buildings [35] because it provides information about on textural data of the materials constituting the various objects. Furthermore, the state of decay can be investigated detecting thermal irregularities. This technology can be integrated with automation to get enhancements of industrial indoor and outdoor inspection of infrastructure and increase quality in production. An overview of possible industrial and non-industrial applications is reported in [36].

An example of the outcome for detection of electrical component in industrial environment is given in Figure 8. In particular, thermal imaging camera may give information about poor connection and internal damage, internal fuse damage, incorrectly secured connection for electrical/electronic components in industrial environment. 
In order to control the robot operations internal sensors have been considered to verify the status of the system with relation of the environment. The display of the internal sensor suite is shown in Fig. 9a) and main characteristics of the sensors are given in Figure 9b).

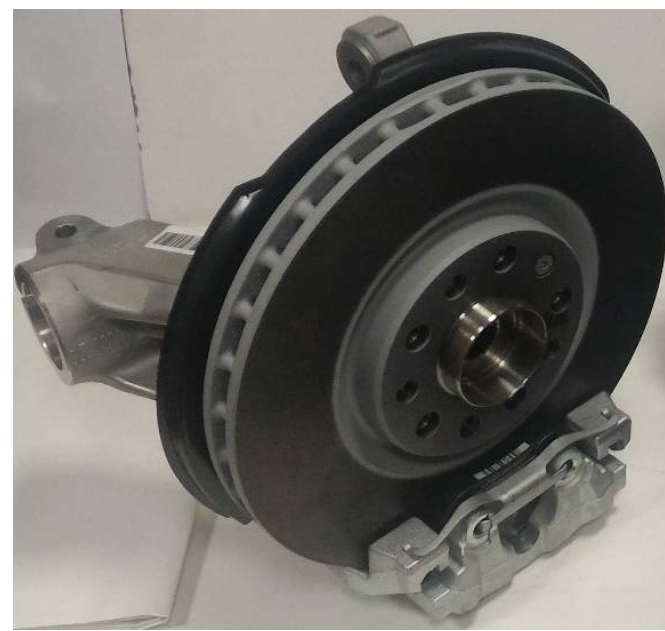

a)

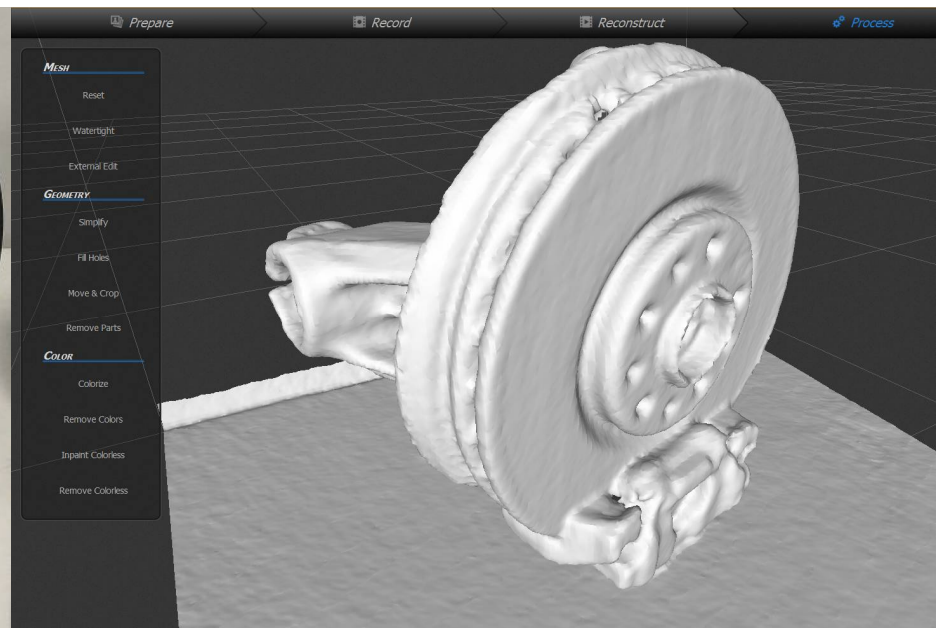

b)

Figure 7. Example of the 3D scan: a) mechanical component: b) StL file of the 3D Scan.

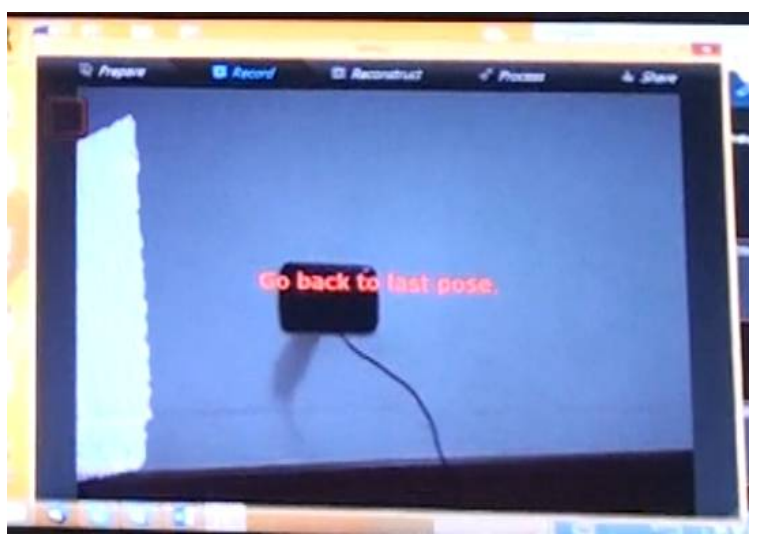

a)

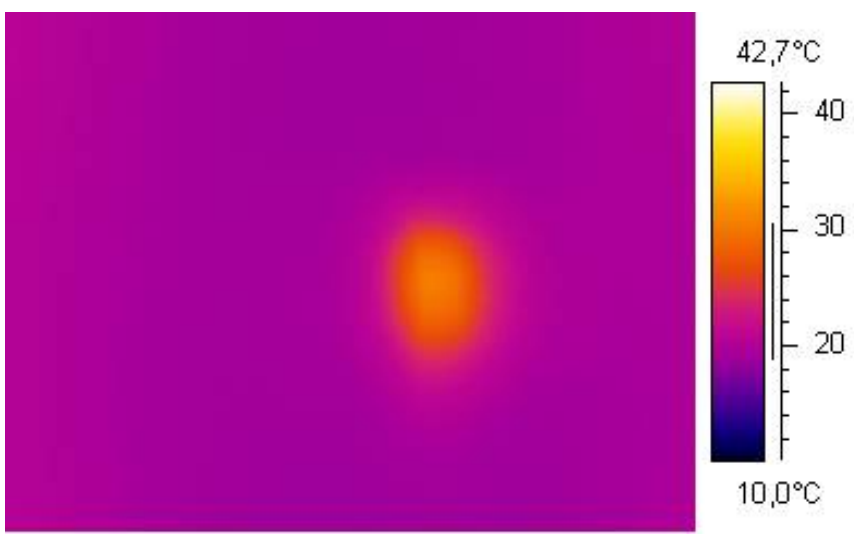

b)

Figure 8. Example of the thermal infrared detection: a) electrical component; b) infrared image.

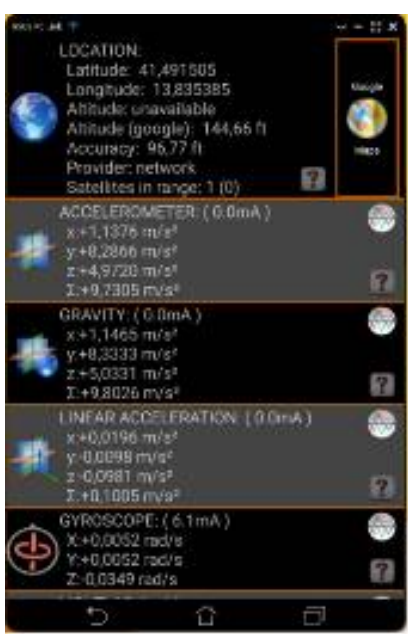

a)

\begin{tabular}{|c|c|c|c|}
\hline Type & Range & Resolution & Power \\
\hline $\begin{array}{c}\text { PSH } \\
\text { Accelerometer } \\
\text { (Intel Inc.) }\end{array}$ & $0 \ldots 39.227$ & $0.01(0.024 \%)$ & $0.006 \mathrm{~mA}$ \\
\hline $\begin{array}{c}\text { PSH Gyroscope } \\
\text { sensor (Intel Inc.) }\end{array}$ & $0 \ldots 34.907$ & $0.002(0.005 \%)$ & $6.1 \mathrm{~mA}$ \\
\hline $\begin{array}{c}\text { PSH Gravity sensor } \\
\text { (Intel Inc.) }\end{array}$ & $0 \ldots 19.613$ & $0.005(0.024 \%)$ & $0.006 \mathrm{~mA}$ \\
\hline $\begin{array}{c}\text { PSH Magnetic field } \\
\text { sensor (Intel Inc.) }\end{array}$ & $0 . .800$ & $0.5(0.062 \%)$ & $0.1 \mathrm{~mA}$ \\
\hline $\begin{array}{c}\text { PSH Linear } \\
\text { Acceleration } \\
\text { sensor (Intel Inc.) }\end{array}$ & $0 \ldots 19.613$ & $0.005(0.024 \%)$ & $0.006 \mathrm{~mA}$ \\
\hline
\end{tabular}

b)

Figure 9. Electronic board with sensors: a) display; b) technical data. 
The sensors on the electronic board are used to understand the status of the robot during teleoperation, as based on gyroscope and tri-axial measurements to prevent overthrow with possible damage to the equipment or loss of the robot control.

\subsection{Communication tools}

Depending upon the application, the operation of the robot can be performed in three ways namely pure teleoperation, safeguarded teleoperation, and autonomous navigation. The last one is out of the scope of the paper and the pure teleoperation is adopted in this context. For safety reasons or simply for convention, it is necessary/preferable that the operator of the robot should be located at a distance; in some cases, wireless communication is possible, in other cases (i.e. thickness of the walls, or unfriendly environment for transmitting and receiving radio waves) wired solution has to be adopted.

In industrial environment, wireless communication is available and preferable in almost all cases to avoid wired solutions that limit the range of mobility and distance of the robot. Indeed, the communication facility consists of: I) Integration of sensors with wireless communication and embedded computing to form smart sensors and smart sensor network with powerful function; II) multi-sensing-function sensors with wireless communication, and advanced data processing and possibility to store on a Cloud.

The sensors information required to navigation and specifically related to the inspection is sent either to the laptops and can be stored on a Cloud [31].

The integration of sensors and its management has been a subject of research activity in different specific domains, as for example related also to monitoring of earthquakes [37], master and slave operations [38], home safety [39] and service robots and devices home-care [40]. It is worth to mention that the IRS involved in industrial sites may also take profit by already developed networks. An industrial network laboratory prototype has been proposed in [41] in which several kits have been implemented for local and remote control issues. Therefore, communications protocols can be also used for remote control and data storage, when the security of network access is also taken into account limiting and controlling the access [41].

If a cable communication system is required due to specific requirements, i.e. nuclear plants, it is necessary to design and operate the robot taking into account the presence of the wired communication of all data, which may greatly limit the robots' operations. An effective and successful solution was adopted in [20] considering a dual-robot system, one devoted to the exploration and one used for carrying the cable drum and unreeling mechanism.

\section{Performance assessment}

The THROO hybrid robot has been equipped with the described sensors and tested in indoor laboratory environment to simulate industrial environmental operating conditions, as it is shown in Figure 10.

Preliminary tests performed on the robotic mobile hybrid platform were reported in [27], in which a comparison was performed between numerical and experimental results giving a good match. In particular, the ability of the THROO hybrid robot was experimentally tested in overpass obstacles. A step field pallet was first simulated and then used for experimental activity. Several tests have been carried out in indoor environment with the on board equipment, as the one reported in Figure 10. In particular, the scan of a wall is shown identifying the electronic component functionality.

More specifically the interoperability of sensors and wireless communication is demonstrated by the photo sequence in Fig.10, in which the robot is tele-operated by wireless communication. The operation of the developed control scheme architecture allows controlling simultaneously internal sensors for navigation and external equipment for 3D scan and thermal imagery. Data is in real-time displayed and stored. 

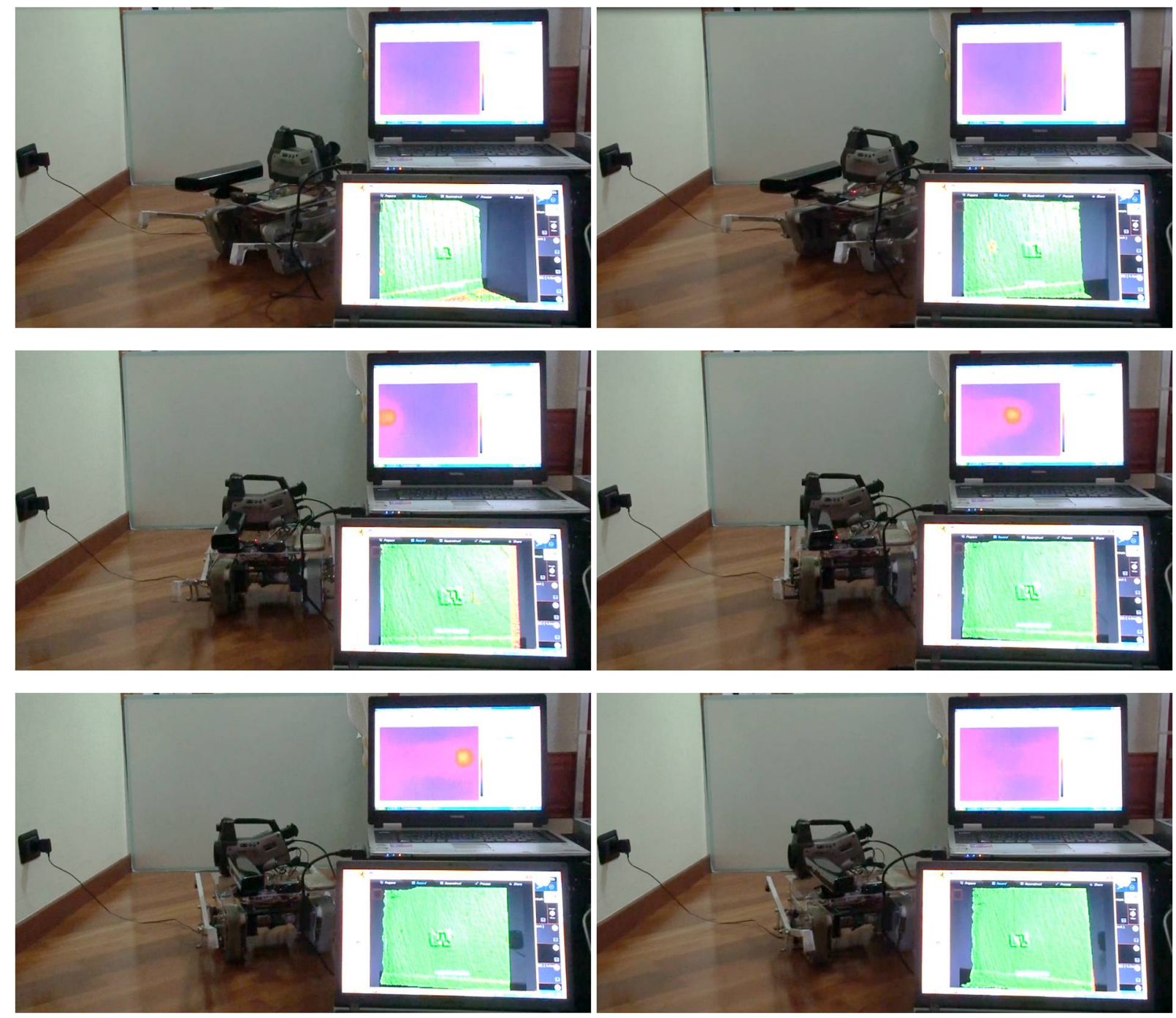

Figure 10. Photo sequence of the experimental test to simulate industrial environment in which the robot is commanded by wireless communication for scanning a wall.

In the reported example in Fig. 10, an electrical element was analyzed in tele-operation as illustrative example during the inspection. Figure 11a) shows a snapshot of the component; Fig. 11c) shows a zoomed view for the 3D relief of the inspected area of about $1 \mathrm{mq}$ and consisting of 57975 vertices and 111670 faces. The thermal image of the component is given in Fig. 11b). All acquired data were monitored on two screens for better visualization, and stored in a PC for data analysis and further reconstruction.

An integration of the sensors data relies on the complete 3D and thermal mapping of objects by giving to the operator an exhaustive set of information, to be further analyzed and processed for decision-making procedures. Moreover, the use of this IRS potentially gives the possibility to obtain set of data from sites dangerous or difficult to access, and simultaneous data acquisition from different sources in one survey.

The IRS then provides a tool for obtaining simultaneously and in real-time data from different types of sensors, with low-cost solution, to be used in industrial environment, in dangerous or inaccessible sites as well. Moreover, the low-cost philosophy for the design allows the possibility to consider a dual or multiple IRS allowing fast substitution if one robot is damaged or lost.

It is worth noting that in the case of poor lighting a led light can be considered, together with a front infrared camera. In addition, a cover is considered that prevents sensors from dust, humidity, and damages. It is worth noting that the cover is not displayed to emphasize the sensors suite and their real time acquisition, as it reported in the photo sequence of Figure 10. 


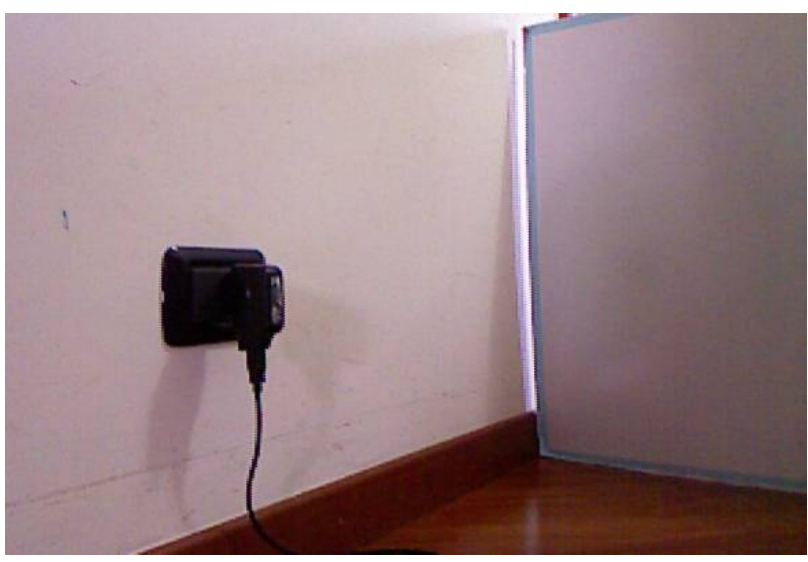

a)

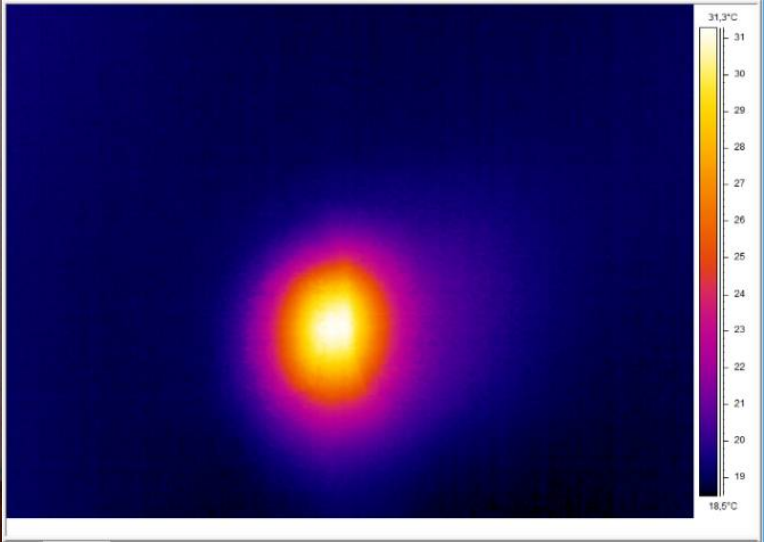

b)

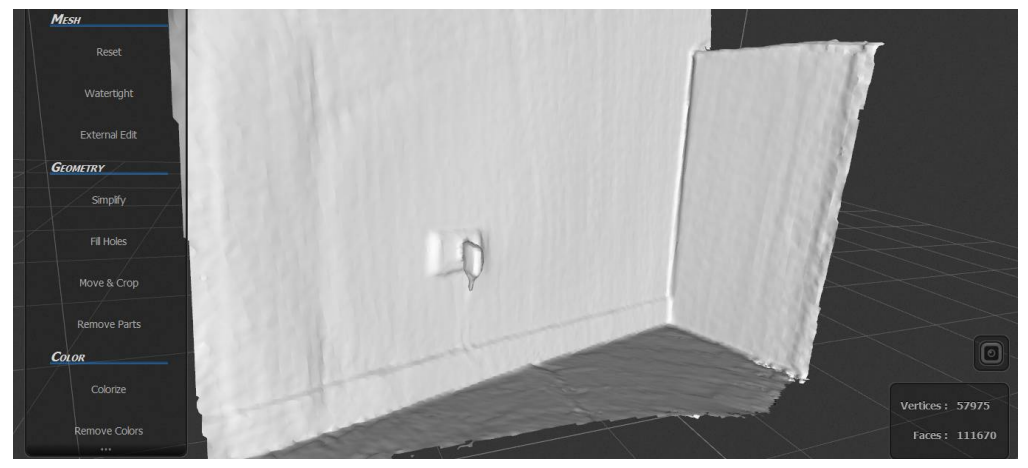

c)

Figure 11. Outcome of the experimental test in Fig.10 for the simultaneous data acquisition of:

a) an electronic component; b) the thermal image of the component; c) the 3D relief.

\section{Conclusion}

In this paper, we have presented a solution for monitoring and inspection of sites based on a hybrid mobile robot equipped with suitable sensors. At the current state, the proposed solution allows real time-multiple data acquisition and storage, which can be further processed for decision-making. More specifically, the integration of sensors with wireless communication and embedded computing, form a smart sensor tool kit and a smart sensor network with powerful functions, which is the final goal of the IRS. Moreover, the development of a tool kit based on a multi- sensing-function sensors, wireless communication with the possibility of storing the data on a Cloud may constitute a solution for a broad range of scenarios. Our project's aim was to test low-cost technologies to monitor industrial sites trying to significantly reduce the acquisition and maintenance costs so as to facilitate wider use of the monitoring systems to widespread their possible applications. More specifically, the hybrid mobile robot (legged and tracked) and the 3D mapping, color information and thermal images are acquired simultaneously to reduce the time needed for a relief and to get a set of information, which can be further processed and integrated for decision-making. In fact, starting from industrial site, for which the system has been originally designed, it can be used for exploration of dangerous or inaccessible sites, brownfields, historical sites, and further extended to outdoor environment.

\section{References}

[1] C. Balaguer, R. Montero, J. G. Victores, S. Martínez, A. Jardón, "Towards Fully Automated Tunnel 
Inspection: A Survey and Future Trends", The 31st International Symposium on Automation and Robotics in Construction and Mining ISARC, 2014.

[2] F. Yao, G. Shao, R. Takaue, A. Tamaki, "Automatic concrete tunnel inspection robot system", Advanced Robotics, 17 (4), p. 319-337, 2003.

[3] F. Zhuang, C. Zupan, Z. Chao, Z. Yanzheng, "A cable-tunnel inspecting robot for dangerous environment" International Journal of Advanced Robotic Systems, 5 (3), p. 243-248, 2008.

[4] A. Bekhit, A. Dehghani, R. Richardson, " Kinematic Analysis and Locomotion Strategy of a Pipe Inspection Robot Concept for Operation in Active Pipelines ,Jnl. of Mechanical Engineering and Mechatronics, 1(1), 2012.

[5] Y. Zhang, G. Yan, "In-pipe inspection robot with active pipe-diameter adaptability and automatic tractive force adjusting", Mechanism and Machine Theory, 42 (12), p. 1618-1631, 2007.

[6] Solo Robots by RedZone, web: http://www.redzone.com/ 2017.

[7] L. Mateos, Marcos Rodriguez y Dominguez, Markus Vincze: "Automatic In-Pipe Robot Centering from 3D to 2D Controller Simplification"; in: "Proceedings of the IEEE/RSJ International Conference on Intelligent Robots and Systems, 2013.

[8] L. Matthies, Y. Xiong, R. Hogg, D. Zhu, A. Rankin, B. Kennedy, M. Hebert, R. Maclachlan, C. Won, T. Frost, G. Sukhatme, M. McHenry and S. Goldberg, "A portable, autonomous, urban reconnaissance robot," Rob.

Autonom. Syst. 40, pp. 163-172, 2002.

[9] D. Wettergreen, S. Moreland, K. Skonieczny, "Design and Field Experimentation of a Prototype Lunar Prospector", the Int. Jnl. of Robotics Research, Vol.29, No.12, pp. 1550-1564, 2010.

[10] T. Aoki, Y.Murayama, S. Hirose, "Mechanical Design of Three-Wheeled Lunar Rover; "Tri-Star IV", IEEE Int. Conf. Rob and Autom., Shangai, pp. 2198-2203, 2011.

[11] A. Seeni, B. Schafer, B. Rebele, N. Tolyarenko, "Robot mobility concepts for extraterrestrial surface exploration", Proceedings of the IEEE Aerospace Conference, pp. 1-14, 2008.

[12] G. Granosik, J. Borenstein, M.G. Hansen, 2007, "Serpentine Robots for Industrial Inspection and Surveillance." Industrial Robotics - Programming, Simulation and Applications, edited by Low Kin Huat. Published by pro-Literatur Verlag, Germany, pp. 633-662, 2007.

[13] I. Maurtua, L. Susperregi, A. Fernández, C. Tubío, C. Perez, J. Rodríguez, T.Felsch, M. Ghrissi, “MAINBOT - mobile robots for inspection and maintenance in extensive industrial plants", Energy Procedia, 49, pp. 1810 - 1819, 2014.

[14] PETROBOT project, web http://petrobotproject.eu/ 2017.

[15] P.E. Sandin, Robot Mechanisms and Mechanical Devices Illustrated, McGraw-Hill, 2003.

[16] R. Siegwart, I. R. Nourbakhsh, Introduction to Autonomous Mobile Robots, MIT Press, 2004.

[17] A. Gonzalez Rodriguez, A. Gonzalez Rodriguez, P. Rea, "A new articulated leg for mobile robots", J. Industrial Robot, 38 (5), pp.521 - 532, 2011.

[18] U. Saranli, M. Buehler, D.E. Koditschek, "RHex: A Simple and Highly Mobile Hexapod Robot", Int. Jnl. of Robotics Research, 20 (7), pp. 616-631, 2001.

[19] E. Ottaviano, P. Rea, "Design and operation of a 2DOF leg-wheel hybrid robot. Jnl of Robotica, Vol. 31, p. 1319-1325, doi: 10.1017/S0263574713000556, 2013.

[20] K. Nagatani, S. Kiribayashi, Y. Okada, K. Otake, K. Yoshida, S. Tadokoro, T. Nishimura, T. Yoshida, E. Koyanagi, M. Fukushima, S. Kawatsuma, "Emergency response to the nuclear accident at the Fukushima Daiichi Nuclear Power Plants using mobile rescue robots". Jnl. Field Robotics, Vol. 30, pp. 44-63, 2013.

[21] B. Yamauchi, P. Rudakevych, "Griffon: A Man-Portable Hybrid UGV/UAV", Industrial Robots, Vol. 31, No. 5, pp. 443-450, 2004.

[22] R. Siegwart, P. Lamon, T. Estier, M. Lauria, R. Piguet, "Innovative Design for Wheeled Locomotion in Rough Terrain", Jnl. of Robotics and Autonomous Systems, Vol. 40, pp. 151-162, 2002.

[23] J. M. García, J. L. Martínez, A. Mandow, A. García-Cerezo, "Caster-leg aided maneuver for negotiating surface discontinuities with a wheeled skid-steer mobile robot", Robotics and Autonomous Systems, Vol. 91, pp. 25-37, 2017.

[24] L. Bruzzone, P. Fanghella, "Mantis: hybrid leg-wheel ground mobile robot", Industrial Robot: An International Journal, Vol. 41 (1), pp. 26 - 36, 2014.

[25] L. Bruzzone and G. Quaglia, "Review article: locomotion systems for ground mobile robots in unstructured environments", Mechanical Sciences, Vol. 3 (2), pp. 49-62, 2012.

[26] ROBOPEC website, available at http://www.robopec.com/ , 2017. 
[27] E. Ottaviano, P.Rea, G. Castelli, "THROO: a Tracked Hybrid Rover to Overpass Obstacles", Advanced Robotics, 28(10), pp. 683-694, 2014.

[28] T. Itul, D., Pisla, "On the kinematics and dynamics of 3-DOF parallel robots with triangle platform", Journal of Vibroengineering, 11(1), pp. 188-200, 2009.

[29] J. Borràs, F. Thomas, E. Ottaviano, M. Ceccarelli, " A reconfigurable 5-DoF 5-SPU parallel platform" Proc. of the 2009 ASME/IFToMM Int. Conf. on Reconfigurable Mechanisms and Robots, ReMAR, Article number 5173892, pp. 617-623, 2009.

[30] E. Ottaviano, S. Vorotnikov, M. Ceccarelli, P. Kurenev, "Design Improvements and Control of a Hhybrid Walking Robot", Robotics and Autonomous Systems, 59 (2), pp.128-141, 2011.

[31] K.-M. Chao, A.E. James, A.G. Nanos, J.-H. Chen, S.-D. Stan, I. Muntean, G. Figliolini, P. Rea, C.B. Bouzgarrou, P. Vitliemov, J. Cooper, J. Van Capelle, "Cloud E-learning for Mechatronics: CLEM", Future Generation Computer Systems. Vol.48, pp. 46-59, 2015.

[32] Y. Takeda, N. Aoyama, T. Tanaami, S. Mizumi, H. Kamata, "Study on the Indoor SLAM Using Kinect", Chapter, Advanced Methods, Techniques, and Applications in Modeling and Simulation, Vol. 4 of the series Proceedings in Information and Communications Technology, Springer Japan, pp 217-225, DOI 10.1007/9784-431-54216-2_24, 2012.

[33] H. Sankrit, B. J. Panwala, P. M. Chandrashekhar, G. Patil, "Indoor SLAM using Kinect Sensor", IJSTE International Journal of Science Technology \& Engineering, Vol. 2, Issue 10, 2016, ISSN (online): 2349-784X [34] FLIR website available at: http://www.flir.com/automation/display/?id=64052, 2017

[35] A. Pelliccio, E. Ottaviano, P. Rea, "Digital and Mechatronic Technologies Applied to the Survey of Brownfields", Chapter 27 in Handbook of Research on Emerging Digital Tools for Architectural Surveying, Modeling, and Representation, IGI Global Eds, 813-829, 2015.

[36] J. Styron, "IR imaging for machine vision and process control", available at: http://www.automation.com/pdf_articles/flir/IR_Imaging_for_Machine_Vision_and_Process_Control.pdf 2017.

[37] M. Ceccarelli, E. Ottaviano, M. Galvagno, "A 3-DOF Parallel Manipulator as Earthquake Motion Simulator", Proc. of the 7th Int. Conf. on Control, Automation, Robotics and Vision ICARCV 2002, Singapore, pp. 944-949, 2002.

[38] M. Sorli, G. Figliolini, S. Pastorelli, P. Rea, P., "Experimental identification and validation of a pneumatic positioning servo-system", Power Transmission and Motion Control, PTMC 2005, Bath, Pages 365-378, 2005. [39] C.-C. Tseng, C.-L. Lin, B.-Y. Shih, C.-Y. Chen, "SIP-enabled Surveillance Patrol Robot", Robotics and Computer Integrated Manufacturing", Vol. 29, pp. 394-399, 2013.

[40] R. Borja, J.R. de la Pinta, A. Álvarez, J.M. Maestre, "Integration of service robots in the smart home by means of UPnP: A surveillance robot case study", Robotics and Autonomous Systems, Vol. 61, pp. 153-160, 2013.

[41] C.P. Leão, F.O. Soares, J.M. Machado, E. Seabra H. Rodrigues, "Design and development of an industrial network laboratory", Int. Jnl of Emerging Technologies in Learning, 6, Special Issue 2, pp. 21-26, 2011. 